\title{
APPLICATIONS OF AFFINE ROOT SYSTEMS TO THE THEORY OF SYMMETRIC SPACES ${ }^{1}$
}

\author{
BY LAWRENCE CONLON
}

Communicated by Raoul Bott, November 25, 1968

Introduction. Let $\left(G ; K_{1}, K_{2}\right)$ be a compact symmetric triad in the sense of [3], $G$ simply connected. The natural action of $K_{1}$ on $G / K_{2}$ is of interest because it is variationally complete [5]. In [3] we introduced certain "affine root systems" in order to describe the orbits of this $K_{1}$-action, and in the present note we wish to announce the classification [4] of these systems and to indicate further applications to the theory of symmetric spaces.

1. Preliminaries. Let $\mathfrak{g}$ be a complex semisimple Lie algebra, $\nu$ an automorphism of $g$, and set $g_{\nu}=\{X \in g: \nu(X)=X\}$. The following is due essentially to de Siebenthal [7] (cf. also [4, \$7]).

(1.1) Proposition. If $\mathfrak{h}_{\nu} \subset \mathfrak{g}_{\nu}$ is a Cartan subalgebra, there is a unique Cartan subalgebra $\mathfrak{h} \subset \mathfrak{g}$ such that $\mathfrak{h}_{\nu} \subset \mathfrak{h}$. There is a finite family a $=\left\{\zeta: \mathfrak{h}_{p} \rightarrow \boldsymbol{C} / \boldsymbol{i Z}\right\}$ of affine functionals and an orthogonal direct sum decomposition

$$
\mathfrak{g}=\mathfrak{h} \oplus \sum \mathfrak{g}_{\mathfrak{s}}, \quad \zeta \in \mathfrak{a}
$$

where $\operatorname{dim}\left(g_{5}\right)=1$ and

$$
\nu \circ \exp (\operatorname{ad}(Z)) \mid g_{\xi}=\exp (2 \pi \zeta(Z)),
$$

for all $Z \in \mathfrak{h}$, and $\zeta \in \mathfrak{a}$. $\zeta(0)$ is pure imaginary for all $\zeta \in \mathfrak{a}$.

$\mathfrak{h}_{\nu}=V \oplus i V$ where $V$ is the real subspace on which the "linear parts" $\tilde{\omega}=\omega-\omega(0)$ of the elements $\omega \in \mathfrak{a}$ are real. One defines

$$
\mathfrak{A}=\{\tilde{\omega} \mid V-i \omega(0): \omega \in \mathfrak{a}\}
$$

interpreted as a set of affine functionals $V \rightarrow R / Z$. This is the system defined by de Siebenthal.

$\mathfrak{g}=\mathfrak{g}_{*} \oplus i \mathfrak{g}_{*}$ where $\mathfrak{g}_{*}$ is the compact real form of $\mathfrak{g}$. Let $s_{1}$ and $s_{2}$ be involutive automorphisms of $\mathrm{g}_{*}, \sigma_{1}$ and $\sigma_{2}$ the extensions of these to anti-involutions of $g$. There correspond symmetric subalgebras $\mathfrak{l}_{1}, \mathfrak{l}_{2}$ of $\mathfrak{g}_{*}$ and noncompact real forms $\mathfrak{g}_{1}, \mathfrak{g}_{2}$ of $\mathfrak{g}$.

Let $\mathfrak{m} \subset g_{*}$ be the simultaneous -1 eigenspace of $s_{1}$ and $s_{2}$. Set $\nu=\sigma_{1} \sigma_{2}$ and choose $\mathfrak{h}_{\nu}$ as in (1.1), but such that $\mathfrak{h}_{\nu} \cap(\mathfrak{m} \oplus i \mathfrak{m})$ is maxi-

\footnotetext{
${ }^{1}$ Research partially supported by NSF GP-6330.
} 
mal abelian in $\mathfrak{m} \oplus i \mathfrak{m}$. Let $\sigma$ denote $\sigma_{1}\left|g_{p}=\sigma_{2}\right| g_{v}$. Note that $\sigma(V)=V$ and that $\sigma$ induces a permutation $\sigma_{*}$ of $\mathfrak{A}$. The pair $\left(\mathfrak{A}, \sigma_{*}\right)$ will be called the affine $\sigma$-system associated to $\left(\mathfrak{g} ; \mathfrak{g}_{1}, g_{2}\right)$ (or to $\left(g_{*} ; \mathfrak{l}_{1}, \mathfrak{l}_{2}\right)$ ).

If we let $V^{-}$denote the +1 eigenspace of $\sigma \mid V$ and $\mathscr{A}^{-}$the set of nonconstant restrictions of elements of $\mathfrak{A}$ to $V^{-}$, we obtain the affine root system of [3].

2. Equivalences and classification. One defines isomorphism $\left(\mathfrak{A}, \sigma_{*}\right) \cong\left(\mathfrak{H}^{\prime}, \sigma_{*}^{\prime}\right)$ via linear isometries $\phi: V \rightarrow V^{\prime}$ carrying $\mathfrak{H}^{\prime} \rightarrow \mathfrak{U}$ and such that $\phi \circ \sigma=\sigma^{\prime} \circ \phi$, and one similarly defines affine equivalence $\left(\mathfrak{Y}, \sigma_{*}\right) \sim\left(\mathfrak{H}^{\prime}, \sigma_{*}^{\prime}\right)$ via affine isometries $\phi: V \rightarrow V^{\prime}$ with $\phi \circ \sigma=\sigma^{\prime} \circ \phi$. Isomorphism $\left(\mathfrak{g} ; \mathfrak{g}_{1}, g_{2}\right) \cong\left(\mathfrak{g} ; \mathfrak{g}_{1}^{\prime}, g_{2}^{\prime}\right)$ is defined via an automorphism $\theta$ of $\mathfrak{g}$ leaving $\mathfrak{g}_{*}$ invariant such that $\theta\left(\mathfrak{g}_{j}\right)=\mathfrak{g}_{j}^{\prime}, j=1,2$. Affine equivalence $\left(g ; g_{1}, g_{2}\right) \sim\left(g ; g_{1}^{\prime}, g_{2}^{\prime}\right)$ means that there are inner automorphisms $\zeta_{1}$, $\zeta_{2}$ of $g$ leaving $g_{*}$ invariant such that $\left(g ; g_{1}, g_{2}\right) \cong\left(g ; \zeta_{1}\left(g_{1}^{\prime}\right), \zeta_{2}\left(g_{2}^{\prime}\right)\right)$.

(2.1) TheOREM. Let $\left(\mathfrak{g} ; \mathfrak{g}_{1}, \mathfrak{g}_{2}\right)$ and $\left(\mathfrak{g} ; \mathfrak{g}_{1}^{\prime}, \mathfrak{g}_{2}^{\prime}\right)$ have respective affine $\sigma$-systems $\left(\mathfrak{A}, \sigma_{*}\right)$ and $\left(\mathfrak{H}^{\prime}, \sigma_{*}^{\prime}\right)$. Then $\left(\mathfrak{g} ; \mathfrak{g}_{1}, \mathfrak{g}_{2}\right) \cong\left(\mathfrak{g} ; \mathfrak{g}_{1}^{\prime}, \mathfrak{g}_{2}^{\prime}\right) \Rightarrow\left(\mathfrak{A}, \sigma_{*}\right)$ $\cong\left(\mathfrak{H}^{\prime}, \sigma_{*}^{\prime}\right) \Rightarrow\left(\mathfrak{g} ; \mathfrak{g}_{1}, \mathfrak{g}_{2}\right) \cong\left(\mathfrak{g} ; \mathfrak{g}_{w(1)}^{\prime}, \mathfrak{g}_{w(2)}^{\prime}\right)$ for a suitable permutation wo of $\{1,2\}$. Likewise, $\left(\mathfrak{g} ; \mathfrak{g}_{1}, \mathfrak{g}_{2}\right) \sim\left(\mathfrak{g} ; \mathfrak{g}_{1}^{\prime}, \mathfrak{g}_{\mathfrak{2}}^{\prime}\right) \Rightarrow\left(\mathfrak{A}, \sigma_{*}\right) \sim\left(\mathfrak{A}^{\prime}, \sigma_{*}^{\prime}\right) \Rightarrow\left(\mathfrak{g} ; \mathfrak{g}_{1}, \mathfrak{g}_{2}\right)$ $\sim\left(g ; g_{w(1)}^{\prime}, g_{w(2)}^{\prime}\right)$.

The affine $\sigma$-systems for all triads $\left(g ; g_{1}, g_{2}\right)$ have been classified up to affine equivalence [4].

3. Topological applications. Consider the action of $K_{1}$ on $G / K_{2}$ as in the introduction. Let $T \subset G / K_{2}$ be the flat geodesic torus described in [3] and [6]. Then $T$ meets orthogonally every $K_{1}$-orbit and $V^{-}$ identifies in a natural way with the universal covering of $T$. The system $\mathfrak{A}^{-}$- describes the singular set in $T$ relative to the $K_{1}$-action [3] and enables us to apply the theory of [2]. If $N \subset G / K_{2}$ is a $K_{1}$ orbit, Theorem 3.1 of [3] shows that the space $\Omega\left(G / K_{2} ; x, N\right)$ of paths on $G / K_{2}$ from the point $x$ to the submanifold $N$ has no torsion in homology iff a certain "regularity" condition $[3$, p. 236] is satisfied by $\mathfrak{A}-$. As a result of [4] we can list up to affine equivalence (and a permutation of $\{1,2\})$ the triads $\left(\mathfrak{g}_{*} ; \mathfrak{l}_{1}, \mathfrak{l}_{2}\right)$ for which $\mathfrak{A}-$ is regular. For $\mathrm{g}_{*}$ simple these are given in the following list.

Type A. $\left(A_{r} ; A_{q} \times A_{r-q-1} \times R, A_{k} \times A_{r-k-1} \times R\right),\left(A_{2 r-1} ; D_{r}, A_{2 r-q} \times R\right)$, $\left(A_{2 r} ; B_{r}, A_{2 r-1} \times R\right),\left(A_{2 r-1} ; C_{r}, C_{r}\right),\left(A_{2 r-1} ; C_{r}, D_{r}\right),\left(A_{2 r-1} ; C_{r}\right.$, $\left.A_{q} \times A_{2 r-q-2} \times R\right),\left(A_{2 r-1} ; D_{r}, A_{1} \times A_{2 r-8} \times R\right)$.

Type B. $\left(B_{r} ; D_{r}, D_{r}\right),\left(B_{r} ; D_{r}, B_{q} \times D_{r-q}\right)$.

Type C. $\left(C_{r} ; C_{q} \times C_{r-q}, C_{k} \times C_{r-k}\right),\left(C_{r} ; C_{q} \times C_{r-q}, A_{r-1} \times R\right)$.

Type D. $\left(D_{r} ; B_{r-1}, B_{r-1}\right),\left(D_{r} ; A_{r-1} \times R, A_{r-1} \times R\right),\left(D_{r} ; D_{r-1} \times R\right.$, $\left.D_{k} \times D_{r-k}\right)$ where $r>k \geqq 1,\left(D_{2 r+k} ; D_{r} \times D_{r+k}, A_{r-1} \times R\right)$ where $k \geqq 0$, 
$\left(D_{r} ; B_{r-1}, D_{k} \times D_{r-k}\right)$ where $r>k \geqq 1,\left(D_{r} ; A_{r-1} \times R, B_{k} \times B_{r-k-1}\right)$ where $r>k \geqq 1,\left(D_{4} ; B_{3}, \omega\left(B_{3}\right)\right),\left(D_{4} ; B_{3}, \omega\left(B_{1} \times B_{2}\right)\right)$. Here $\omega$ is the triality automorphism of $D_{4} ; B_{3}$ and $B_{1} \times B_{2}$ are standardly imbedded in $D_{4}$.

Type E. $\left(E_{6} ; D_{5} \times R, D_{5} \times R\right),\left(E_{6} ; F_{4}, F_{4}\right),\left(E_{6} ; F_{4}, C_{4}\right),\left(E_{6} ; D_{5} \times R\right.$, $\left.A_{5} \times A_{1}\right),\left(E_{6} ; F_{4}, D_{5} \times R\right),\left(E_{6} ; F_{4}, A_{5} \times A_{1}\right),\left(E_{7} ; E_{6} \times R, E_{6} \times R\right)$, $\left(E_{7} ; A_{7}, E_{6} \times R\right),\left(E_{7} ; E_{6} \times R, D_{6} \times A_{1}\right)$.

Type F. $\left(F_{4} ; B_{4}, B_{4}\right),\left(F_{4} ; B_{4}, C_{3} \times A_{1}\right)$.

4. Commuting involutions. Following Hermann [6] one asks whether there is an inner automorphism $\zeta$ of $g$ leaving $\mathfrak{g}_{*}$ invariant such that $\zeta \sigma_{1} \zeta^{-1}$ commutes with $\sigma_{2}$. Using (1.1) and (2.1) one can prove the answer is affirmative iff $\left(\mathfrak{A}, \sigma_{*}\right) \sim\left(\mathfrak{U}^{\prime}, \sigma_{*}^{\prime}\right)$ where $\phi \in \mathfrak{Y}^{\prime}$ implies $\phi(0)=0$ or $\frac{1}{2}$.

As Hermann has shown [6, Proposition 2.1], the existence of totally geodesic $K_{1}$-orbits in $G / K_{2}$ is completely bound up with the solutions $\zeta$ to this problem. The system $\left(\mathfrak{A}, \sigma_{*}\right)$ somewhat clarifies this situation as we now indicate.

Let $p: V^{-} \rightarrow T$ be the natural covering map. Supposing that the commuting involutions problem has a solution, we lose no generality in assuming $\sigma_{1} \sigma_{2}=\sigma_{2} \sigma_{1}$ (hence $s_{1} s_{2}=s_{2} s_{1}$ ). Then if $\Lambda$ is the lattice $\left\{X \in V^{-}: \phi(X)=0\right.$ or $\frac{1}{2}$, all $\left.\phi \in \mathfrak{U}\right\}$, we have the following.

(4.1) Proposition. $\Sigma=p(\Lambda)$ is the subset of $T$ consisting of the points whose $K_{1}$-orbits are totally geodesic in $G / K_{2}$.

The assumption $s_{1} s_{2}=s_{2} s_{1}$ implies that $s_{1}$ defines an involutive isometry (again called $s_{1}$ ) of $G / K_{2}$. This situation is quite general.

(4.2) Proposition. Let $G$ be simply connected. Then every involutive isometry of $G / K_{2}$ having nonempty fixed point set is conjugate (in the isometry group) to one produced by an involutive automorphism $s_{1}$ of $G$ commuting with $s_{2}$.

We explicitly identify the fixed point set of the involution $s_{1}$ in $G / K_{2}$. For each $\phi \in \mathfrak{A}-$, let $\tilde{\phi}$ be the linear part as in $\$ 1$ and define $h_{\phi} \in V^{-}$by $h_{\phi} \perp \operatorname{Ker}(\tilde{\phi})$ and $\tilde{\phi}\left(h_{\phi}\right)=2$. The lattice $\Lambda_{\epsilon}$ spanned by these vectors $h_{\phi}$ is exactly $p^{-1}\left(\left\{K_{2}\right\}\right)$.

(4.3) ThEOREM. Again assume $G$ simply connected and $s_{1} s_{2}=s_{2} s_{1}$. Let $\Lambda_{*}=\frac{1}{2} \Lambda_{0}$ and $\Sigma_{*}=p\left(\Lambda_{*}\right)$. Then $\Sigma_{*} \subset \Sigma$ and the fixed point set of $s_{1}$ in $G / K_{2}$ is exactly the union of the $K_{1}$-orbits of the elements of $\Sigma_{*}$.

5. Pseudo-Riemannian symmetric spaces. The explicit solutions of the commuting involutions problem make possible a classification of the isomorphism classes of those $\left(g ; g_{1}, g_{2}\right)$ for which $\sigma_{1} \sigma_{2}=\sigma_{2} \sigma_{1}$. For 
each of these $\left(g_{1}, g_{1} \cap g_{2}\right)$ and $\left(g_{2}, g_{1} \cap g_{2}\right)$ are dual pseudo-Riemannian symmetric pairs [1]. All pseudo-Riemannian pairs may be obtained in this way; hence [4] contains implicitly the classification [1].

In the following, $\Re=\{\phi \in \mathfrak{X}: \phi(0)=0\}$ and $\mathfrak{R}^{-}=\left\{\phi \in \mathfrak{A}^{-}: \phi(0)=0\right\}$. These are identified as subsets of the dual spaces $V^{*}$ and $\left(V^{-}\right)^{*}$ respectively. For other terminology in the theorem below, cf. [1].

(5.1) TheOREM. Let $\mathrm{g}$ be simple, $\sigma_{1} \sigma_{2}=\sigma_{2} \sigma_{1}$. The corresponding dual symmetric pairs are either both reducible or both irreducible. They are reducible iff $\Re^{-}$spans a subspace of $\left(V^{-}\right)^{*}$ of codimension one, and in this case the dual pairs are mutually isomorphic. They are irreducible iff $\mathfrak{R}^{-}$spans $\left(\mathrm{V}^{-}\right)^{*}$. The dual symmetric pairs are either both complex symmetric or both fail to be so. They are complex symmetric iff $\Re$ spans a subspace of $V^{*}$ of codimension one and $\Re^{-}$spans $\left(V^{-}\right)^{*}$. In this case the dual pairs are actually semikählerian.

These facts are proven without classification.

\section{REFERENCES}

1. M. Berger, Les espaces symetriques non compacts, Ann. Sci. Ecole Norm. Sup. 74 (1957), 85-177.

2. R. Bott and H. Samelson, Applications of the theory of Morse to symmetric spaces, Amer. J. Math. 80 (1958), 964-1029.

3. L. Conlon, The topology of certain spaces of paths on a compact symmetric space, Trans. Amer. Math. Soc. 112 (1964), 228-248.

4. - Classification of affine root systems and applications to the theory of symmetric spaces, Mimeographed Notes, Washington University, St. Louis, Mo., 1968.

5. R. Hermann, Variational completeness for compact symmetric spaces, Proc. Amer. Math. Soc. 11 (1960), 544-546.

6. - Totally geodesic orbits of groups of isometries, Nederl. Akad. Wetensch. Proc. Ser. A 65 (1962), 291-298.

7. J. de Siebenthal, Sur les groupes de Lie compacts non connexes, Comment. Math. Helv. 31 (1956) 41-89.

Washington University, St. Louis, Missouri 63130 and

St. Louis University, St. Louis, Missouri 63103 